\title{
Viral Laryngitis
}

National Cancer Institute

\section{Source}

National Cancer Institute. Viral Laryngitis. NCI Thesaurus. Code C27305.

Acute inflammation of the larynx caused by viruses, including rhinovirus, influenza virus, parainfluenza virus, and adenovirus. 